\section{Allergenquelle Maus}

n der Gruppe der felltragenden Tiere

weisen auch Mäuse eine hohe Allergenität auf - festgestellt zunächst bei Beschäftigten in Tierlaboratorien und -farmen, die mit Mäusen arbeiteten. Eine ähnlich hohe Allergenbelastung wie in der Tierhaltung wurde jetzt in innerstädtischen Wohnungen in Baltimore im USBundesstaat Maryland festgestellt. Man hatte 100 Schlafzimmer von Asthmakindern untersucht und in der Luft Mus-m1-Spiegel in Konzentrationen festgestellt, die bei sensibilisierten Personen Asthmaanfälle auslösen können. Assoziiert war das Vorkommen des Maus-Hauptallergens mit Essensresten auf dem Fußboden sowie Löchern und Rissen in der Wand.

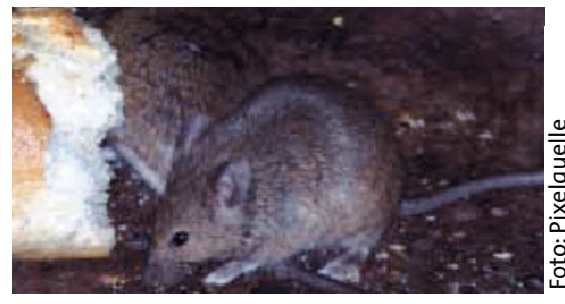

Einen tendenziell schützenden Effekt hatte die Anwesenheit einer Katze in der Wohnung - die Katzenhaltung wird aber nicht als gesundheitsfördernde Maßnahme für die Kinder empfohlen. Vielmehr sollten die Wohnungen ordentlich saubergemacht und die Löcher verschlossen werden, empfehlen die Autoren. $\quad b k$

Matsui EC et al. J Allergy Clin Immunol 2005; 115: 358-63

\title{
Kontaktallergie gegen Budesonid
}

S ie ist selten, kommt aber doch vor: eine Kontaktdermatitis gegen inhalative Glukokortikoide. Französische Dermatologen beschrieben jetzt den Fall einer Mutter, die ihrem 3-jährigen asthmakranken Sohn per Babyhaler Budesonid und Terbutalin applizierte. Ab dem 4. Behandlungstag zeigten sich bei der Frau einige Stunden nach der Glukokortikoidapplikation bei ihrem Sohn geschwollene und juckende Läsionen im Gesicht, begleitet von einer Konjunktivitis. Die daraufhin verordnete Gluko-

kortikoidcreme (Desonid) verschlechterte den Hautbefund. Der Hauttest bestätigte dann den Verdacht auf eine Kontaktallergie gegen Budesonid. Positive Hautreaktionen zeigten sich auch auf Desonid und Triamcinolonacetonid - beides wie Budesonid Glukokortikoide der Gruppe B. Tests mit Glukokortikoiden aus anderen Gruppen verliefen dagegen negativ.

Raison-Peyron $\mathbf{N}$ et al. Ann Dermatol

Venereol 2005; 132: 143-6

\section{Hypnose hilft bei Heuschnupfen}

A

llergische Erkrankungen weisen oft eine psychosomatische Komponente auf. Ob diese Komponente durch Hypnose zu beeinflussen ist, untersuchte jetzt ein Forscherteam vom Universitätshospital Basel. Dazu wurden 79 Patienten mit allergischer Rhinitis in zwei Gruppen aufgeteilt. Die erste Gruppe erlernte eine Autosuggestionstechnik, um in einen tranceähnlichen Zustand zu gelangen. Dabei sollten sich die Probanden auf allergenfreie Umgebungen, wie zum Beispiel eine Winterszenerie mit glitzernden Eiskristallen, konzentrieren. Die zweite Gruppe diente als Kontrolle. Tatsächlich hatten sich nach einer Pollensaison in der
Hypnosegruppe sowohl Symptom- als auch Medikationsscore im Vergleich zur Kontrolle signifikant gebessert ( $\mathrm{p}<0,001)$, auch war die subjektive Lebensqualität gestiegen. Als in der folgenden Pollensaison auch die Kontrollgruppe die Hypnosetechnik anwenden durfte, verschwanden die beobachteten Unterschiede wieder. Die Autoren berichten, dass die Selbsthypnose oft schon nach wenigen Minuten zu einem Rückgang der allergischen Beschwerden führte, und empfehlen ihre Methode als adjuvante Therapie. es

Langewitz W et al. Psychother Psychosom 2005; $74: 165-72$

\section{Wanderschraube}

_ in 52-jähriger Mann suchte seinen Zahnarzt wegen eines prothetischen Eingriffs auf. Während der Behandlung verlor der Zahnarzt eine Schraube und konnte sie nicht mehr in der Mundhöhle auffinden. Der Patient war beschwerdefrei und suchte daher nur auf Anraten des Zahnarztes eine Nothilfe auf, um eine Röntgenaufnahme des Thorax anfertigen zu lassen. Die Schraube fand sich in einem peripheren Bronchus der rechten Lunge (Abb. oben). Bei der zunächst starren, dann mit dem Fiberskop durchgeführten Bronchoskopie unter Narkose bei Spontanatmung konnte die Schraube nicht aufgefunden werden. Der Patient brauchte sehr lange, um nach Beendigung der Narkose wieder aufzuwachen, und entwickelte einen starken Husten, sodass ein Inhalationssyndrom vermutet wurde.
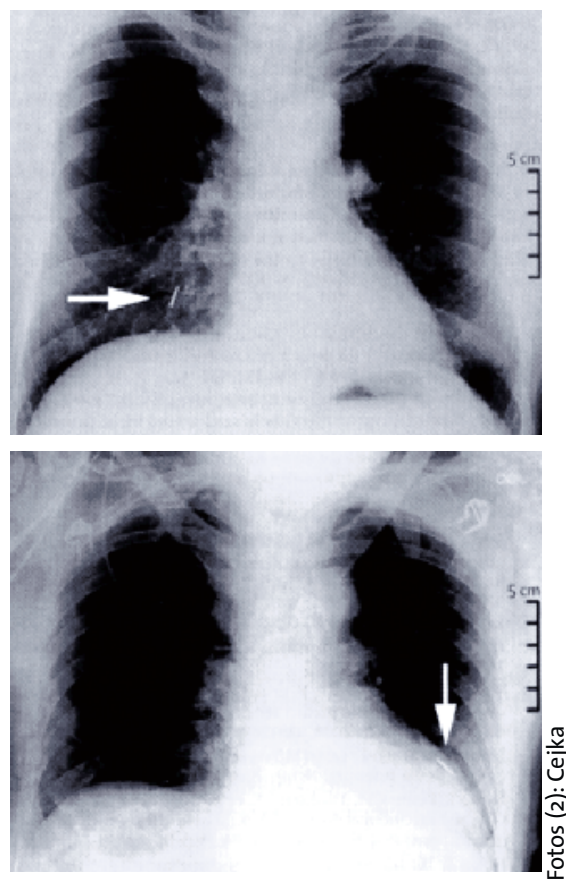

Eine zweite Röntgenaufnahme des Thorax ergab zum Erstaunen aller Beteiligten, dass die Schraube nun in einem Bronchus der linken Lunge lag (Abb. unten). Am nächsten Tag konnte der Fremdkörper problemlos in einer zweiten Bronchoskopie entfernt werden. Wann genau die Schraube die Seite gewechselt hatte, war nicht mehr zu klären.

hsf

Cejka JC, Gautier G. Lancet 2005; 365: 680 